\title{
Análise comparativa entre os índices tomográficos em pacientes com ceratocone inicial
}

\author{
Comparative analysis of tomographic indices in patients at early stage of keratoconus
}

Francisco Wellington Rodrigues ${ }^{1}$ (D), Ana Beatriz Vieira Vilela² (D), Julia Faustino Nishi ${ }^{(0)}$, Rodrigo Egídio da Silva ${ }^{1}$ ()

Descritores:

Topografia da córnea; Córnea; Doenças da córnea; Ceratocone; Lesões da córnea; Transplante de córnea

Keywords: Corneal topography; Cornea; Corneal diseases; Keratoconus; Corneal injuries; Corneal transplantation

Recebido: 22/11/2020

Aceito: 24/4/2021

Autor correspondente: Rodrigo Egídio da Silva Avenida Americano do Brasil, 260 - Setor CEP: 74180-110 - Goiânia (GO), Brasil. E-mail: rodrigoegidio@ver.med.br

Instituição de realização do trabalho: VER Hospital de Olhos, Goiânia, GO, Brasil.

Fonte de auxílio à pesquisa: não financiado.

Conflitos de interesse: os autores declaram que não há conflitos de interesses.

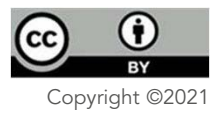

\section{RESUMO}

Objetivo: Avaliar sensibilidade do volume corneano, asfericidade e índice D para o diagnóstico precoce de ceratocone.

Métodos: Estudo transversal analítico, realizado entre dezembro de 2018 e outubro de 2020, no qual foram analisados os prontuários de 39 pacientes (78 olhos) diagnosticados com ceratocone por um único subespecialista em córnea, por meio dos critérios de Belin/Ambrosio Enhanced Ectasia. Os dados coletados foram: asfericidade anterior, asfericidade posterior, índice $\mathrm{D}$ e suas frações $(\mathrm{Da}, \mathrm{Db}$, Dt, Dp e Df). Os dados foram digitados e manipulados em Excel, para posterior tratamento utilizando o programa Statistical Package for Social Science do Windows, versão 21.0. As variáveis categóricas foram apresentadas como valor absoluto e percentual. Variáveis contínuas foram apresentadas como média \pm desvio-padrão e mediana (intervalo de confiança de 95\%).

Resultados: Foram analisados 78 olhos, com idade média de $28,2 \pm 5,8$ anos e porcentagem entre o sexo feminino e masculino de $59,0 \%$ e $41,0 \%$, respectivamente. O índice D apresentou estreita relação com sua fração Db nos casos iniciais de ceratocone, enquanto a fração Dt apresentou tal relação de forma reduzida.

Conclusão: A correlação entre o índice $\mathrm{D}$ e sua fração $\mathrm{Db}$ sugere alteraçães precoces na elevação corneana posterior, colaborando para o diagnóstico de ceratocone subclínico.

\section{ABSTRACT}

Objective: To assess sensitivity of corneal volume, corneal asphericity, and D index in early diagnosis of keratoconus.

Methods: A cross-sectional analytical study, carried out between December 2018 and October 2020, which analyzed the medical records of 39 patients (78 eyes) diagnosed as keratoconus by a single corneal specialist, using the criteria of Belin-Ambrosio Enhanced Ectasia Display. The collected data were anterior asphericity, posterior asphericity, $\mathrm{D}$ index and its parameters ( $\mathrm{Da}, \mathrm{Db}, \mathrm{Dt}, \mathrm{Dp}$ and $\mathrm{Df})$. The data were entered and handled in Excel, for later data treatment using the software Statistical Package for Social Science, version 21.0, for Windows. The categorical variables were presented as absolute value and percentages. The continuous variables were presented as mean \pm standard deviation, and median (95\% of confidence interval).

Results: We analyzed 78 eyes, mean age of $28.2 \pm 5.8$ years, $59.0 \%$ of sample were female, and $41.0 \%$, male. The $\mathrm{D}$ index showed a close relation to its $\mathrm{Db}$ parameter in the cases of keratoconus at early stage, while Dt parameter showed a reduced relation.

Conclusion: The correlation between the D index and its Db parameter suggests early changes in the posterior corneal elevation, contributing to early diagnosis of subclinical keratoconus. 


\section{INTRODUÇÃO}

O ceratocone é uma doença corneana degenerativa, progressiva, bilateral e assimétrica, responsável por alterações na superfície da córnea. ${ }^{(1)}$ Caracteriza-se por afinamento central, protusão apical e astigmatismo irregular, ocasionando redução da acuidade visual. ${ }^{(2)}$ Sua etiologia ainda é indeterminada, mas sua associação com doenças atópicas, retardo mental, trauma ocular por lente de contato e doenças sistêmicas do colágeno já é conhecida. Classicamente, inicia-se na puberdade e progride até a quarta década de vida, quando geralmente se estabiliza. ${ }^{(3)}$

O diagnóstico é feito por exame clínico e avaliação tomográfica da córnea. Os sinais tomográficos da doença incluem: aumento da curvatura anterior da córnea, astigmatismo irregular e assimétrico, afinamento estromal e aumento da curvatura posterior. ${ }^{(4)}$ O Pentacam ${ }^{\circledR}$ High Resolution (HR), tomógrafo do seguimento ocular anterior comumente usado no diagnóstico do ceratocone, escaneia as superfícies anterior e posterior da córnea por meio da câmara de Scheimpflug rotacional. O aparelho mede 25 mil pontos de elevação e, a partir daí, com um modelo tridimensional do segmento anterior, obtém-se um cálculo preciso de todos os sinais característicos da doença. Ao final do exame, as imagens são transferidas para o computador, no qual são analisadas por meio de um software. ${ }^{(5)}$

$\mathrm{O}$ índice $\mathrm{D}(\mathrm{BAD}-\mathrm{D})$, que é fracionado em $\mathrm{Da}, \mathrm{Db}$, Dt, Dp e Df é uma variável analisada na progressão da ectasia corneana. ${ }^{(6)}$ Os valores de D representam desvios da média significativos para a progressão paquimétrica da superfície corneana; Da representa desvios paquimétricos da média significativos para a superfície anterior da córnea; Db representa desvios paquimétricos da média significativos da superfície posterior da córnea; Dt representa desvios paquimétricos da média em relação ao ponto mais fino da córnea; Dp representa desvios da média da progressão paquimétrica da córnea; Df representa desvios paquimétricos da média em relação ao deslocamento do ponto mais fino da córnea. ${ }^{(6)}$

Este manuscrito tem por objetivo analisar a sensibilidade do índice $\mathrm{D}$ e suas frações no diagnóstico precoce do ceratocone. O estudo deve contribuir para a identificação precoce da doença e auxiliar tanto o oftalmologista geral e/ou específico na elaboração de estratégias terapêuticas específicas, de acordo com o grau da doença, sendo estas menos traumáticas e eficazes nos estágios iniciais do ceratocone.

\section{MÉTODOS}

Trata-se de estudo do tipo transversal analítico, realizado em hospital da rede privada de Goiânia (GO). O estudo, que corresponde a uma vertente de outro projeto, obteve aprovação do Comitê de Ética em Pesquisa do Hospital das Clínicas da Universidade Federal de Goiás, sob CAAE: 70173617.0.0000.5083.

Foram analisados 39 pacientes (78 olhos) diagnosticados com ceratocone em estágios iniciais pelo mesmo especialista em córnea por meio dos critérios de Belin/ Ambrosio Enhanced Ectasia, ${ }^{(7)}$ utilizando-se o tomógrafo Pentacam HR 70900 (Oculus Wetzlar, Alemanha).

Os critérios de Belin/Ambrosio(7) avaliam raio de curvatura anterior (A); raio de curvatura posterior (B); paquimetria da córnea no ponto mais fino $(\mathrm{C})$; melhor acuidade visual corrigida (D); presença de cicatriz que permite a visualização da íris (+); cicatriz que não permite a visualização da íris (++) ou ausência de cicatriz (-). A partir dessa avaliação, classifica-se o paciente entre os estágios zero e quatro, sendo os casos subclínicos classificados como zero e os casos extremos, como quatro.

Adotaram-se como objeto de estudo as seguintes variáveis: asfericidade anterior, asfericidade posterior, índice D e suas frações ( $\mathrm{Da}, \mathrm{Db}, \mathrm{Dt}$, Dp e Df). Estas, obtidas de prontuários eletrônicos, foram coletadas a partir de três exames de tomografia corneana subsequentes, separados por um intervalo de tempo igual ou maior que 6 meses.

Foram incluídos pacientes diagnosticados com ceratocone pelos critérios de Belin/Ambrosio Enhanced Ectasia e idade igual ou superior a 18 anos. Não parcitiparam do estudo aqueles com histórico de cirurgias ou doenças oftalmológicas prévias e dados incompletos no prontuário.

\section{Análise estatística}

Os dados foram digitados e manipulados em Excel, para posterior tratamento utilizando o programa Statistical Package for Social Science (SPSS), versão 21.0, do Windows.

As variáveis categóricas foram apresentadas como valor absoluto (f) e percentual (\%). As variáveis contínuas foram apresentadas como média \pm desvio-padrão, mediana (intervalo de confiança de 95\%).

Inicialmente foi realizada uma análise descritiva de todas as variáveis do estudo.

A fim de verificar a existência de correlação entre o índice D e os índices Df, Db, Dp, Dt e Da, nos olhos direito e esquerdo, foi usada a análise de regressão linear, uma vez que se trata de variáveis contínuas. O teste foi feito dois a dois, sendo índice D x índice Df, índice D x índice Db, índice $\mathrm{Dx}$ índice $\mathrm{Dp}$, índice $\mathrm{D} x$ índice Dt e índice $\mathrm{D} x$ índice 
Da. Essas correlações foram realizadas para o olho direiro e para o olho esquerdo.

Para o teste foi considerado nível de 95\% de confiança, ou seja, p < 0,05 foi considerado significativo.

\section{RESULTADOS}

O estudo foi composto de 39 pacientes (78 olhos), com idade média de $28,2 \pm 5,8$ anos e porcentagem entre o sexo feminino e masculino de 59,0\% e 41,0\%, respectivamente. Do total de 78 olhos, 11 foram excluídos, por não atenderem aos critérios de inclusão.

Na tabela 1, observa-se a média dos índices D e suas subunidades nos olhos direito e esquerdo. Ao analisar o índice $\mathrm{D}$ por meio de regressão linear da média dos três exames realizados, observou-se, tanto em olho direito quanto em esquerdo, a correlação direta entre as frações $\mathrm{Da}, \mathrm{Db}, \mathrm{Df}$ e Dp no valor final do índice $\mathrm{D}$, destacando-se $\mathrm{Db}$ pela maior correlação, quando comparado aos outros índices. O Dt, por sua vez, apresentou menor correlação com o valor final do índice $\mathrm{D}$ em ambos os olhos (Tabela 2).

Tabela 1. Parâmetros das médias do índice D e suas subunidades

\begin{tabular}{lll}
\hline Medidas & \multicolumn{2}{c}{ Parâmetros } \\
\hline Olho direito & & \\
índice D & $3,56 \pm 2,58$ & $2,37(2,63-4,49)$ \\
Df & $2,51 \pm 2,92$ & $1,76(1,45-3,56)$ \\
Db & $2,04 \pm 3,07$ & $0,44(0,93-3,15)$ \\
Dp & $3,98 \pm 5,59$ & $2,24(1,96-6,00)$ \\
Dt & $1,40 \pm 1,36$ & $1,26(0,91-1,89)$ \\
Da & $1,85 \pm 0,92$ & $1,83(1,52-2,19)$ \\
Olho esquerdo & & \\
Índice D & $3,26 \pm 2,04$ & $2,63(2,56-3,97)$ \\
Df & $1,83 \pm 2,51$ & $1,36(0,98-2,70)$ \\
Db & $1,41 \pm 2,25$ & $0,74(0,56-2,25)$ \\
Dp & $3,17 \pm 2,95$ & $2,33(2,16-4,18)$ \\
Dt & $0,91 \pm 1,02$ & $1,08(0,51-1,30)$ \\
Da & $1,82 \pm 0,82$ & $1,88(1,54-2,11)$ \\
\hline IC95\%. intervaliana &
\end{tabular}

Tabela 2. Análise de regressão linear do índice D (média das três medidas)

\begin{tabular}{|c|c|c|c|}
\hline Medidas & $\mathbf{R}^{2}$ & B & Valor de $p$ \\
\hline \multicolumn{4}{|c|}{ Olho direito } \\
\hline Df & 0,491 & 0,847 & $<0,001^{*}$ \\
\hline $\mathrm{Db}$ & 0,929 & 0,942 & $<0,001^{*}$ \\
\hline $\mathrm{Dp}$ & 0,782 & 0,363 & $<0,001^{*}$ \\
\hline $\mathrm{Dt}$ & 0,439 & 1,116 & $<0,001^{*}$ \\
\hline $\mathrm{Da}$ & 0,881 & 2,487 & $<0,001^{*}$ \\
\hline \multicolumn{4}{|c|}{ Olho esquerdo } \\
\hline Df & 0,334 & 0,519 & $0,001^{*}$ \\
\hline $\mathrm{Db}$ & 0,882 & 0,814 & $<0,001^{*}$ \\
\hline $\mathrm{Dp}$ & 0,835 & 0,560 & $0,001^{*}$ \\
\hline $\mathrm{Dt}$ & 0,014 & 0,221 & 0,546 \\
\hline $\mathrm{Da}$ & 0,799 & 2,15 & $<0,001^{*}$ \\
\hline
\end{tabular}

significativo

$\mathrm{R}^{2}$ : coeficiente de correlação; B: inclinação da reta; p: probabilidade.
A comparação entre olho direito e olho esquerdo, pelo teste de Wilcoxon, não revelou significância estatística e clínica entre as variáveis asfericidade anterior, asfericidade posterior e índice D, Da, Db, Dt, Dp e Df.

\section{DISCUSSÃO}

O ceratocone é uma doença corneana caracterizada por alterações anatômicas que resultam em redução progressiva da acuidade visual e podem ser avaliadas por meio da tomografia corneana. Esse exame, que corresponde ao padrão-ouro para avaliação do segmento anterior, revela, por meio de índices, uma visão global das características corneanas. ${ }^{(8)}$ Apesar disso, a identificação dos casos subclínicos permanece um desafio, já que a especificidade e a sensibilidade de tais índices pode encontrar-se reduzida. ${ }^{(9)}$

Ao considerar o índice D como um dos parâmetros mais preditivos de evolução da ectasia corneana, ${ }^{(10)}$ dois achados destacam-se neste estudo. Observa-se estreita correlação identificada entre Db e índice D, de forma que, assumindo Db como uma medida das variações da superfície corneana posterior, tal achado confirma a fisiopatologia do ceratocone que evidencia uma alteração precoce da elevação corneana posterior nos casos subclínicos. ${ }^{(11)}$

Por outro lado, a reduzida correlação entre Dt e índice D, sugere que as variações do ponto mais fino da córnea teriam pouca influência sobre índice D nas fases iniciais da ectasia corneana. No entanto, sabe-se, tanto pelo estudo da doença, quanto por múltiplos estudos, ${ }^{(12,13)}$ que o afilamento verificado na paquimetria é determinante para a progressão e a identificação do ceratocone. É válido ressaltar que o resultado elucidado possivelmente relaciona-se com a amostra analisada, uma vez que, no diagnóstico precoce, o ponto mais fino da córnea não estaria significativamente alterado. ${ }^{(14)}$

No âmbito sociodemográfico, observou-se concordância em relação à ectasia corneana quanto à faixa etária descrita na literatura: entre 25 e 35 anos. ${ }^{(15)} \mathrm{A}$ despeito da maior prevalência no sexo feminino encontrada, não há registros de predileção por gênero na ectasia corneana. ${ }^{(4)}$

Quantos às limitações deste estudo, podem-se identificar a quantidade de olhos avaliados e o direcionamento para uma única variável utilizada no diagnóstico de ceratocone, o índice D. A contribuição, portanto, está na realização de estudos mais amplos acerca das variáveis utilizadas para o diagnóstico do ceratocone, especialmente o índice D e sua fração Db. Embora tal índice não seja o único parâmetro para avaliação da doença, é possível que se destaque como sinal de alerta para alterações da ectasia corneana precoce. 


\section{CONCLUSÃO}

Observou-se uma estreita relação entre o índice D e sua fração Db em casos iniciais de ceratocone, concordando com a literatura e com a fisiopatologia da doença. Por esse motivo, recomenda-se a realização de maiores estudos sobre a relação entre as variáveis e os estágios iniciais da ectasia corneana.

\section{AGRADECIMENTOS}

A todos os profissionais do VER Hospital de Olhos, pelo suporte e pela assistência prestados durante a execução deste trabalho, e em especial à funcionária Rosângela Santos, pela prestabilidade no auxílio da coleta dos dados analisados nesta pesquisa.

\section{REFERÊNCIAS}

1. Miháltz K, Kovács I, Takács Á, Nagy ZZ. Evaluation of keratometric, pachymetric, and elevation parameters of keratoconic corneas with pentacam. Cornea. 2009;28(9):976-80.

2. Uras R, Pavês L. Ceratocone : fatores prognósticos. 2005;68(4):491-4.

3. De Luis Eguileor B, Escudero Argaluza J, Pijoán Zubizarreta Jl, Santamaria Carro A, Etxebarria Ecenarro J. Evaluation of the reliability and repeatability of scheimpflug system measurement in keratoconus. Cornea. 2018;37(2):177-81.

4. Vazirani J, Basu S. Keratoconus: current perspectives. Clin Ophthalmol. 2013;7:2019-30

5. Valbon BF, Salomão MQ, Ramos I, Canedo AL, Nogueira L, Ambrósio Júnior R. Importância da tomografia de córnea para o diagnóstico de ectasia. Rev Bras Oftalmol. 2012;71(5):302-8.
6. Yamane IS. Avaliação de parâmetros tomográficos de córnea e segmento anterior e de variáveis desencadeadas pela resposta ocular à tonometria de não contato [tese]. São Paulo: Universidade de São Paulo, 2012. [citado 2021 Mar 26]. Disponível em: https://www.teses.usp.br/teses/ disponiveis/5/5149/tde-10102012-103707/publico/IrisdeSouzaYamane. pdf

7. Belin MW, Duncan JK. Keratoconus: The ABCD Grading System. Klin Monbl Augenheilkd. 2016;233(6):701-7.

8. Belin MW, Khachikian SS, Ambrósio R, Salomão M. Keratoconus/ectasia detection with the oculus pentacam: Belin/Ambrósio enhanced ectasia display. Highlights Ophthalmol. 2007 [cited 2021 Mar 26].;35(6):5-12 Available from: https://www.oculus.de/uploads/media/belin.pdf

9. Hashemi H, Beiranvand A, Yekta A, Maleki A, Yazdani N, Khabazkhoob M Pentacam top indices for diagnosing subclinical and definite keratoconus. J Curr Ophthalmol. 2016;28(1):21-6.

10. Valbon BF, Santos RT, Ramos I, Canedo AL, Nogueira L, Ambrósio Júnior R. A tomografia de córnea e segmento anterior na propedêutica do exame complementar na avaliação de ectasia. Rev Bras Oftalmol. 2013;72(1):54-8.

11. Muftuoglu O, Ayar O, Ozulken K, Ozyol E, Akıncı A. Posterior corneal elevation and back difference corneal elevation in diagnosing forme fruste keratoconus in the fellow eyes of unilateral keratoconus patients. J Cataract Refract Surg. 2013;39(9):1348-57.

12. Belin MW, Ambrósio R. Scheimpflug imaging for keratoconus and ectatic disease. Indian J Ophthalmol. 2013;61(8):401-6.

13. Schiano-Lomoriello D, Bono V, Abicca I, Savini G. Repeatability of anterior segment measurements by optical coherence tomography combined with Placido disk corneal topography in eyes with keratoconus. Sci Rep. 2020;10(1):1-6.

14. Awad EA, Abou Samra WA, Torky MA, El-Kannishy AM. Objective and subjective diagnostic parameters in the fellow eye of unilateral keratoconus. BMC Ophthalmol. 2017;17(1):186.

15. Mian LC, Carvalho DA, Souza MA, Moraes TA, Costa MI, Moreira GA, et al. Perfil epidemiológico dos pacientes portadores de ceratocone atendidos em um hospital privado de Campo Grande-MS. Ensaios e Ciência: Ciências Biológicas, Agrárias e da Saúde. 2013;17(3):113-21. 